\title{
Historical Views, Conventional Approaches, and Evolving Management Strategies for Myeloproliferative Neoplasms
}

\author{
Brady L. Stein, MD, MHS ; Jason Gotlib, MD ; Murat Arcasoy, MDc; Marie Huong Nguyen, MD; \\ Neil Shah, MD, PhD; ; Alison Moliterno, MD; Catriona Jamieson, MDs; Daniel A. Pollyea, MD, $\mathrm{MS}^{\mathrm{h}}$; \\ Bart Scott, MD; Martha Wadleigh, MD ${ }^{j}$; Ross Levine, MD*; Rami Komrokji, MD'; Rebecca Klisovic, MD ${ }^{\mathrm{m}}$; \\ Krishna Gundabolu, MDn; Patricia Kropf, MD; Meir Wetzler, MDp; Stephen T. Oh, MD, PhD; \\ Raul Ribeiro, MDr; Rita Paschal, MD; Sanjay Mohan, MD'; Nikolai Podoltsev, MD, PhD; \\ Josef Prchal, MDv; Moshe Talpaz, MD'; David Snyder, MDw; Srdan Verstovsek, MDª and \\ Ruben A. Mesa, MDy
}

\section{Abstract}

The classical Philadelphia chromosome-negative myeloproliferative neoplasms (MPN), which include essential thrombocythemia, polycythemia vera, and myelofibrosis (MF), are in a new era of molecular diagnosis, ushered in by the identification of the JAK2 ${ }^{\mathrm{V} 617 \mathrm{~F}}$ and $\mathrm{CMPL}$ mutations in 2005 and 2006, respectively, and the CALR mutations in 2013. Coupled with increased knowledge of disease pathogenesis and refined diagnostic criteria and prognostic scoring systems, a more nuanced appreciation has emerged of the burden of MPN in the United States, including the prevalence, symptom burden, and impact on quality of life. Biological advances in MPN have translated into the rapid development of novel therapeutics, culminating in the approval of the first treatment for MF, the JAK1/JAK2 inhibitor ruxolitinib. However, certain practical aspects of care, such as those regarding diagnosis, prevention of vascular events, choice of cytoreductive agent, and planning for therapies, present challenges for hematologists/oncologists, and are discussed in this article. (J Natl Compr Canc Netw 2015;13:424-434)

From aNorthwestern University Feinberg School of Medicine, Chicago, Illinois; 'Stanford Cancer Institute, Stanford University, Stanford, California; 'Duke Cancer Institute, Duke University, Durham, North Carolina; 'University of Michigan Comprehensive Cancer Center, Ann Arbor, Michigan; eUniversity of California, San Francisco, San Francisco, California; $f$ Johns Hopkins University School of Medicine, Baltimore, Maryland; 'Moores Cancer Center, UC San Diego, San Diego, California; hUniversity of Colorado Cancer Center, Aurora, Colorado; 'Fred Hutchinson Cancer Research Center, Seattle, Washington; 'Dana Farber Cancer Institute, Boston, Massachusetts; ${ }^{k}$ Memorial Sloan Kettering Cancer Center, New York; New York; 'Moffitt Cancer Center and Research Institute, Tampa, Florida; mThe Ohio State University, Columbus, Ohio; nFred \& Pamela Buffett Cancer Center, Omaha, Nebraska; ${ }^{\circ}$ Fox Chase

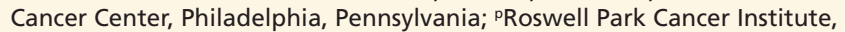
Buffalo, New York; 'Washington University-Siteman Cancer Center, St Louis, Missouri; 'St. Jude Children's Research Hospital, Memphis, Tennessee; sUniversity of Alabama at Birmingham, Birmingham, Alabama; tVanderbilt-Ingram Cancer Center, Nashville, Tennessee; "Yale University School of Medicine; New Haven, Connecticut; vUniversity of Utah, Salt Lake City, Utah; dUniversity of Michigan Comprehensive Cancer Center,

Ann Arbor, Michigan; "City of Hope Cancer Center, Los Angeles, California; xThe University of Texas MD Anderson Cancer Center, Houston, Texas; and yMayo Clinic Cancer Center, Scottsdale, Arizona.

Submitted August 4, 2014; accepted for publication January 5, 2015. Correspondence: Ruben A. Mesa, MD, Mayo Clinic Cancer Center, 13400 East Shea Boulevard, Scottsdale, AZ 85259. E-mail: mesa.ruben@mayo.edu The following authors have no relevant financial relationships to disclose: Drs. Nguyen, Shah, Wadleigh, Levine, Klisovic, Gundabolu, Wetzler, Ribeiro, Paschal, and Mohan.
The following authors have disclosed relationships with commercial interests:

Dr. Stein: Advisory board and speaker's bureau for Incyte Corporation. Advisory board for Sanofi Oncology.

Dr. Gotlib: Advisory board, honoraria, and research funding from Incyte Corporation. Steering committee and research funding from Novartis. Research funding from Gilead Sciences, Inc. and Promedior.

Dr. Arcasoy: Research support from Incyte Corporation and Gilead Sciences, Inc. Dr. Moliterno: Advisory board for Incyte Corporation.

Dr. Jamieson: Research funding from Roche and Johnson \& Johnson Services, Inc.

Dr. Pollyea: Advisory board for and research support from Celgene Corporation.

Dr. Scott: Speaker and advisor for Incyte Corporation.

Dr. Komrokji: Research funding from and advisory board for Incyte Corporation.

Dr. Kropf: Consulting for Takeda Oncology. Speakers bureau for Celgene Corporation.

Dr. Oh: Research support from and consulting for Incyte Corporation and Gilead Sciences, Inc. Research support from Cell Therapeutics.

Dr. Podoltsev: Advisory board for Incyte Corporation.

Dr. Prchal: Advisory board for Gilead Sciences, Inc. and Incyte Corporation. Dr. Talpaz: Research funding from ARIAD Pharmaceuticals, Inc.; BristolMyers Squibb Company; Pfizer Inc.; and Incyte Corporation.

Dr. Snyder: Advisory board for Incyte Corporation.

Dr. Verstovsek: Research support from Incyte Corporation; AstraZeneca Pharmaceuticals LP; Lily Oncology; Roche; Geron Corporation; NS Pharma, Inc.; Bristol-Myers Squibb Company; Novartis; Infinity Pharmaceuticals; YM Biosciences, Inc.; Gilead Sciences, Inc.; Seattle Genetics, Inc.; Promedior, Inc.; Cell Therapeutics; Galena Biopharma; and Pfizer Inc.

Dr. Mesa: Research support from Gilead Sciences, Inc.; Incyte Corporation; Celgene Corporation; Novartis; NS Pharma, Inc.; Promedior, Inc.; and Sanofi. 
Evolving Management Strategies for Myeloproliferative Neoplasms

The classical Philadelphia chromosome-negative myeloproliferative neoplasms (MPN), which include essential thrombocythemia (ET), polycythemia vera (PV), and myelofibrosis (MF), were initially described in the medical literature in 1879, 1892, and 1934, ${ }^{1}$ respectively, and in 1951, Dameshek ${ }^{2}$ speculated on a "myelostimulatory factor" common to these conditions that he classified as "myeloproliferative disorders" (MPD). Then, nearly 55 years after Dameshek's treatise, the JAK2 ${ }^{\mathrm{V} 617 \mathrm{~F}}$ mutation was discovered, representing a watershed moment that renewed research interests in MPN. ${ }^{3}$

Prevalence of MPN in the United States is now better understood, partly based on a recent analysis of 2 large health plans, which showed the prevalence (per 100,000) of ET as 38 to 57; PV as 44 to 57; and MF as 4 to $6 .{ }^{4}$ These data showed the numbers of persons with ET, PV, and MF living in the United States in 2010 to be approximately 134,000, 148,000, and 13,000, respectively. The burden experienced by patients in the United States with MPN has also been better described. An analysis of the SEER-Medicare linked database showed that survival was shortest for patients with MF, but those with ET and PV also had inferior survival rates to matched controls. ${ }^{5}$ In addition, more clarity now exists regarding the impact of the MPN symptom burden, attributed partly to cytokine excess, splenomegaly, hyperviscosity (PV), thrombotic complications, and cytopenias (MF). In some patients with MF, the impairment in quality of life is as or more severe than that observed in patients with metastatic cancer or acute myeloid leukemia. ${ }^{6-8}$ Subsequently, validated tools, such as the MPN Symptom Assessment Form (MPN-SAF), have been created to assess the prevalence and impact of such MPN symptoms. ${ }^{9}$ The ability to assess prognosis is also becoming increasingly sophisticated, especially in MF with expected incorporation of molecular genetic and cytogenetic abnormalities; current tools are used at diagnosis (International Prognostic Scoring System [IPSS] ${ }^{10}$ or during follow-up (Dynamic IPSS [DIPSS], DIPSS plus; Table 1)..11,12

As important, the recognition of JAK-STAT dysregulation has led to the identification of novel therapeutics, culminating in the first approval of a drug for patients with MF, the JAK inhibitor ruxolitinib. This article discusses the rapidly evolving understanding of disease pathogenesis, with an emphasis on driving mutations, and reviews practical aspects in the care of patients with MPN, focusing on diagnostic considerations, thrombosis prevention, supportive care, and therapeutic strategies.

\section{Impact of Molecular Genetic Abnormalities}

The discovery of JAK2 ${ }^{\mathrm{V} 617 \mathrm{~F}}$, and subsequently $\mathrm{MPL}^{\mathrm{W} 515 \mathrm{~L} / \mathrm{K}}$, in $5 \%$ to $10 \%$ of patients with ET or MF clarified the central theme of JAK-STAT dysregulation to MPN pathogenesis. ${ }^{13,14}$ The JAK2 ${ }^{\mathrm{V} 617 \mathrm{~F}}$ mutation is present in $95 \%$ of patients with PV (JAK2 exon 12 mutations are seen in $2 \%-3 \%,{ }^{15}$ and $L N K$ mutations are infrequently identified in JAK2-negative erythrocytosis ${ }^{16}$ ) and in $50 \%$ to $60 \%$ of patients with ET or MF. ${ }^{17}$ More recently, mutations in exon 9 of the calreticulin (CALR) gene were identified in substantial proportions of patients with ET and MF who lacked JAK2 ${ }^{\mathrm{V} 617 \mathrm{~F}}$ or cMPL mutations. ${ }^{18,19}$ In contrast to the initial observations in patients with only ET or MF, CALR mutations were recently described in 2 patients with PV without JAK2 ${ }^{\mathrm{V} 617 \mathrm{~F}}$ mutations. ${ }^{20}$ Approximately $10 \%$ of patients with ET and MF lack JAK2, CALR, or MPL mutations, and have been referred to as being "triple-negative." JAK2 and cMPL mutations were incorporated into the 2008 WHO criteria for ET, PV, and MF, and CALR mutations are similarly expected to be included diagnostic criteria in the next WHO classification. The high prevalence of these clonal markers and others permits reclassification of these diseases as neoplasms in lieu of prior nosology, MPD (Figure 1).

These "driving" mutations also impact prognosis, especially in MF. In a study of 617 patients with $\mathrm{MF}$, the median overall survival was longest in patients with CALR mutations (17.7 years), intermediate-length in patients with MF with JAK2 and MPL mutations (9.2 and 9.1 years, respectively), and shortest in patients considered "triple-negative" (3.2 years). ${ }^{21}$ The cumulative incidence of leukemic transformation was also lowest in patients with CALR mutations (9.4\%) compared with those with JAK2 mutations (19.4\%), MPL mutations (16.9\%), or triple-negative status (34.4\%).

Another study reported the longest median survival (16 years) and lowest rate of blast transformation $(6.5 \%)$ in patients with CALR-mutated MF, especially when compared with patients with JAK2 mutations considered triple-negative. ${ }^{22}$ More specifi- 
Stein et al

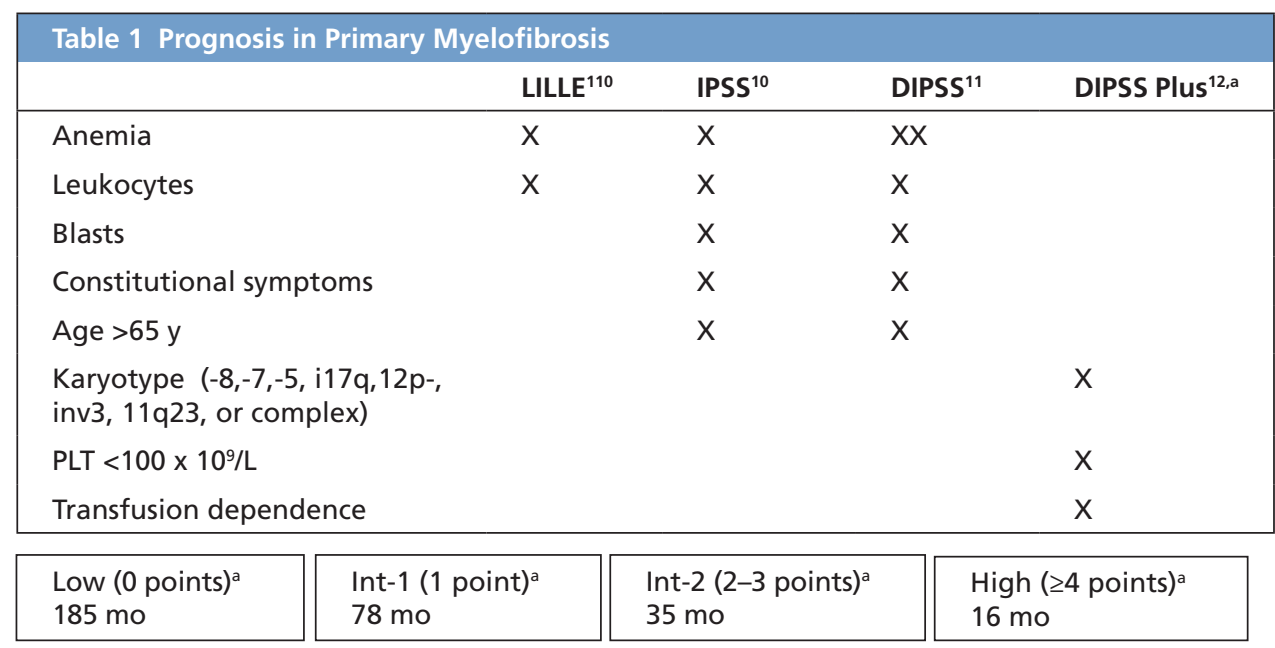

$X$, included variables; $X X$, anemia weighted with 2 points in DIPSS.

IPSS at diagnosis: Low $=0$ points, Int- $1=1$ point; Int- $2=2$ points; High risk $\geq 3$ points.

DIPSS during disease course: Low = 0 points; Int- $1=1-2$ points; Int-2 = 3-4 points; High = 5-6 points.

Abbreviations: DIPSS, Dynamic International Prognostic Scoring System; Int, intermediate; IPSS, International Prognostic Scoring System;

PLT, platelet count.

aDIPSS plus during disease course: Incorporate DIPSS score (Low = 0; Int-1 = 1; Int-2 = 2; High =3) plus added variables, including karyotype

(1 point), thrombocytopenia (1 point), and transfusion dependence (1 point). For example, a patient with advanced age (1 point), anemia ( 2 points), and constitutional symptoms (1 point) would have DIPSS Int-2-risk disease; if transfusion-dependence and deletion of chromosome 8 are considered with DIPSS plus, this patient would have 2 points for the DIPSS score and 2 points (plus variables), for a DIPSS plus score of 4 (high risk).

cally, the prognostic impact of CALR mutations may depend on the type of mutation, because improved outcomes seemed to be restricted to those with type 1 mutations ( 52 base pair deletions) compared with those with type 2 mutations ( 5 base pair insertions). ${ }^{23}$ Other genetic lesions, outside the JAK-STAT pathway, have been identified in patients with MPN, particularly in those with MF, including ASXL1, TET2, IDH1/IDH2, EZH2, TP53, and SRSF2 genes; ASXL1, IDH, EZH2, and SRSF2 mutations have been associated with leukemic evolution and reduced survival time. ${ }^{24}$

\section{From Disorder to Neoplasm: "MPD to MPN"}

\author{
MPN \\ Polycythemia vera \\ Essential thrombocytosis \\ Myelofibrosis \\ Chronic myeloid leukemia \\ Chronic neutrophilic leukemia \\ Chronic eosinophilic leukemia, NOS \\ Systemic mastocytosis \\ MPN, unclassifiable \\ Myeloid (and lymphoid) neoplasms with eosinophilia
}

\section{Diagnostic Challenges}

The MPN molecular markers lack specificity; therefore, consideration of clinical, laboratory, and histologic features is required to define the MPN subtype. Major and minor criteria for diagnosis of the MPN subtype were published previously. ${ }^{25}$ Reactive thrombocytosis and secondary erythrocytosis are more common than ET or PV; MPN markers distinguish primary from secondary causes of abnormal blood counts. Because the JAK2 ${ }^{\mathrm{V} 617 \mathrm{~F}}$ mutation is frequently identified in patients with hepatic (Budd-Chiari syndrome) or portal vein thrombosis, molecular testing should be considered even in the absence of overt MPN features. ${ }^{26}$

\author{
Molecular Genetic Abnormality \\ $J A K 2^{\mathrm{V} 617 \mathrm{~F}}, J A K 2$ exon 12 \\ $J A K 2^{\mathrm{V} 617 F}, C A L R, C M P L$ \\ $J A K 2^{\mathrm{V} 617 F}, C A L R, C M P L$ \\ $B C R-A B L$
CSF3R and SETBP1 mutations
??
KIT $T^{\mathrm{D} 816 \mathrm{~V}}$ \\ ??
}

PDGFRA, PDGFRB, FGFR1

Figure 1 Molecular genetic abnormalities in the myeloproliferative neoplasms.

Abbreviations: MPD, myeloproliferative disorders; MPN, myeloproliferative neoplasms; NOS, not otherwise specified. 
Evolving Management Strategies for Myeloproliferative Neoplasms

In his 1951 treatise, Dameshek ${ }^{2}$ alluded to mimicry inherent to the MPNs; coupled with the potential for phenotypic evolution, distinction of the specific MPN subtype is sometimes difficult. ${ }^{27}$ Distinguishing ET from prefibrotic MF can be challenging but is important, given that the natural history differs; although both can present with an isolated thrombocytosis, overt MF and leukemic transformation occur more frequently in the latter. ${ }^{28}$ Anemia, leukocytosis, and splenomegaly usually favor prefibrotic MF over ET, complementing recognition of such bone marrow features as hypercellularity, granulocytic predominance, and megakaryocyte atypia that also favor prefibrotic MF. ${ }^{29}$ However, not all consider these morphologic criteria for distinction between ET and prefibrotic MF to be reliable or reproducible. ${ }^{30}$ It is also important to recognize early or masked PV, which generally encompasses patients with PV features but without an absolute erythrocytosis (hemoglobin level $>16.5 \mathrm{~g} / \mathrm{dL}$ in women or $>18.5 \mathrm{~g} / \mathrm{dL}$ in men), and to distinguish it from JAK2 ${ }^{\mathrm{V} 617 \mathrm{~F}}$-positive ET. ${ }^{31} \mathrm{Al}$ though PV Study Group criteria relied on red cell mass testing, because of the limited availability of this test, the ideal surrogate for an increased red cell mass is under question, and the hematocrit level rather than the hemoglobin level is being proposed as such a marker. ${ }^{32}$ In fact, hematocrit level $(>48 \%$ in women or $>52 \%$ men) is used by the British Committee for Standards in Haematology (BCSH), and a recent study suggested that these criteria were more adept at defining overt and masked PV compared with WHO criteria using hemoglobin level..$^{33}$ Recent studies suggest that nuclear red cell mass testing, although of limited availability, and bone marrow biopsy may be useful in patients with PV features without absolute erythrocytosis, especially when trying to distinguish JAK2 $2^{\mathrm{V} 617 \mathrm{~F}}$-positive ET from PV. ${ }^{34-36}$ It has been suggested that these MPNs do not exist along a spectrum but are distinct entities, and defining the MPN subtype has therapeutic and prognostic implications. ${ }^{22}$ Recently, to aid in this distinction, experts have proposed lowering the hemoglobin threshold for men and women, and including bone marrow histology as a major criterion for PV diagnosis. ${ }^{37}$

\section{Vascular Risk Assessment}

Clinical management of ET and PV is largely based on thrombosis prevention, given its morbidity and mortality. ${ }^{38-40}$ In ET, the annual rate of thrombosis nears $1.9 \% .^{38}$ Thrombosis rates in contemporary pa- tients with PV are estimated to be $2.6 \%$ to $2.7 \%$ per year. This is lower than the rate of $4.4 \%$ reported previously. ${ }^{39,41,42}$ Prevention is primarily based on predicting vascular risk, which is evolving in ET and now includes the impact of not only advanced age and thrombosis history but also cardiovascular risk factors and presence of the JAK2 ${ }^{\mathrm{V} 617 \mathrm{~F}}$ mutation. ${ }^{38} \mathrm{In}$ $\mathrm{PV}$, the most reproducible risk factors for thrombosis include age older than 60 years and prior thrombosis. The rate of thrombotic events in patients with MF may be comparable to that of those with ET, nearing $1.75 \%$ per year, especially in patients older than 60 years with a JAK2 $2^{\mathrm{V} 617 \mathrm{~F}}$ mutation. Sex may be an additional risk factor for thrombosis, because presentation with abdominal venous thrombosis (AVT) is typically a feature seen in younger women with PV. ${ }^{43}$ Leukocytosis and an increased JAK2 allelic burden are also emerging risk factors for thrombosis. ${ }^{44} \mathrm{~Pa}$ tients with CALR mutations were recently shown to have a lower risk of thrombosis ${ }^{18,19,45,46}$ compared with those with JAK2 $2^{\mathrm{V} 617 \mathrm{~F}}$ mutations. The pathophysiology of MPN-associated thrombosis is additionally influenced by platelet, leukocyte, and endothelial activation, and activated protein $\mathrm{C}$ resistance, inflammation, and circulating microparticles. ${ }^{44}$ The hope is that surrogate markers that capture these derangements will be validated and incorporated into thrombosis risk assessment.

\section{Cytoreductive Therapy}

Goals of cytoreductive therapy include prevention of thrombosis, control of myeloproliferation, and relief of MPN symptoms. ${ }^{47}$ In the United States, hydroxyurea emerged as the most widely used cytoreductive agent, based on lower observed thrombosis rates compared with phlebotomized historical controls and lower observed rates of leukemia or solid malignancies compared with chlorambucil or radioactive phosphorous. ${ }^{48}$ Efficacy in PV is typically extrapolated from randomized controlled trials in patients with ET. In patients with ET at high risk, hydroxyurea was more effective in preventing thrombosis than placebo. ${ }^{49}$ Compared with anagrelide, hydroxyurea was more effective in preventing arterial thrombosis and associated with lower rates of bleeding and myelofibrosis. ${ }^{50}$ Interestingly, mutational status may influence response, because patients with $J A K 2^{\mathrm{V} 617 \mathrm{~F}}$ mutations appeared preferentially sensitive to hy- 
droxyurea over anagrelide. ${ }^{51}$ Use of anagrelide waned after this study, but it was more effective than hydroxyurea in preventing venous thrombosis, because the rate was nearly one-fourth that of hydroxyurea (odds ratio, 0.27; $P=.006$.)..$^{50}$ More recently, a comparison between anagrelide and hydroxyurea in a noninferiority study showed similar rates of major and minor bleeding, thrombosis, and discontinuation in those with WHO-classified ET compared with the PT-1 study, which may have included patients with ET and prefibrotic MF. ${ }^{50,52}$

The association between cytoreductives (especially hydroxyurea) and leukemic transformation is controversial. Among 326 patients with ET treated with cytoreduction, 17 developed leukemia. Of these, 14 were treated with hydroxyurea; a high proportion had $17 \mathrm{p}$ deletions. ${ }^{53}$ Associations with genetic alterations of $17 \mathrm{p}$ (including p53), hydroxyurea exposure, and evolution to MPN blast phase have also been reported in patients with PV. ${ }^{54}$ A long-term follow-up from the French Polycythemia Study Group reported higher-than-expected rates of evolution to acute myeloid leukemia in patients treated with hydroxyurea: $6.6 \%, 16.5 \%$, and $24.2 \%$ at 10,15 , and 20 years, respectively. ${ }^{55}$ Other studies have argued against any association with leukemia and hydroxyurea ${ }^{40,56}$ in ET or $\mathrm{PV},{ }^{42}$ or have shown associations when only chemotherapies were used sequentially. ${ }^{57}$

Lack of association with leukemia, along with improved safety and efficacy, renewed interest in the use of pegylated interferon-alpha in PV and ET. Pegylated interferon-alpha has shown high rates of hematologic response ( $95 \%$ at 12 months) and JAK2 allele burden response (partial 48\%, complete 24\%) in newly diagnosed patients with PV. ${ }^{58}$ However, adverse events were noted in $89 \%$ of patients, and the treatment discontinuation rate was 35\% (24\% because of toxicity). Complete hematologic and molecular responses have also been reported in patients with previously treated ET (77\% and $17 \%$, respectively) and PV (76\% and 18\%, respectively); those who did not experience a complete molecular response were more likely to have mutations outside the JAK-STAT pathway. ${ }^{59}$ Interestingly, in this study, 29\% of patients discontinued therapy but maintained a hematologic remission for a median of 28 months of observation. The MPD Research Consortium is currently conducting studies comparing pegylated interferon and hydroxyurea in high- risk patients with ET and PV, and its use as salvage therapy for patients with hydroxyurea resistance or intolerance or AVT (ClinicalTrial.gov identifiers: NCT01259856 and NCT01259817).

\section{Antiplatelet Therapy, Anticoagulation, Phlebotomy, and Special Clinical Situations}

Additional strategies to reduce the risk of thrombosis include the use of antiplatelet therapies. Use of aspirin in ET is typically extrapolated from a large PV study. In this study of 518 patients with PV, aspirin reduced the risk of a combined end point of nonfatal myocardial infarction (MI), stroke, or death from cardiovascular causes and the risk of a combined end point of nonfatal MI, stroke, pulmonary embolism, major venous thrombosis, or death from cardiovascular causes. ${ }^{39} \mathrm{~A}$ meta-analysis suggested that low-dose aspirin was associated with only a nonsignificant reduction in the risk of fatal thrombosis and all-cause mortality compared with no treatment. ${ }^{60}$ No data support the use of clopidogrel alone or in combination with aspirin. Intriguing in vitro evidence is available to support twice-daily aspirin, ${ }^{61}$ but this approach has not been validated in a clinical trial.

Phlebotomy is a cornerstone of PV therapy, and the hematocrit target of $45 \%$ or less has been established by a randomized trial given the lower risk of cardiovascular events reported when compared with a hematocrit of $45 \%$ to $50 \% .{ }^{62}$ In particular, the cardiovascular event rate was $10.9 \%$ in the higher hematocrit arm (hazard ratio, 2.69; $P=.01$ ) compared with $4.4 \%$ in the lower hematocrit arm, favoring more aggressive control in all prespecified subgroups (gender, advanced age, thrombosis history, platelet and white blood cell cut points, and presence of splenomegaly). In patients with thrombosis, the duration of anticoagulation therapy is not well established, but it is often prescribed indefinitely in those with AVT. ${ }^{47}$ No current data are available on the use of novel oral anticoagulants. Plateletpheresis is a consideration for patients with life-threatening thrombohemorrhagic complications. Strategies for managing MPN in the perioperative period may include normalizing the red blood cell mass for patients with PV and the use of cytoreductive therapy to address thrombocytosis. Because thrombocytosis can increase the hemorrhagic rather than thrombotic tendency, ${ }^{63}$ this becomes an important concern 
Evolving Management Strategies for Myeloproliferative Neoplasms

to communicate to the surgeon; the use of venous thromboembolism prophylaxis after surgery must be individualized, because bleeding and thrombosis rates are increased in this period. ${ }^{64}$ Finally, MPN can present in younger women, and clarifying the use of cytoreductive agents, aspirin, and deep vein thrombosis prophylaxis is important when managing pregnant patients with MPN. Similarly, some reports of pediatric, adolescent, and young adult MPN presentations ${ }^{43,65,66}$ suggest that adult diagnostic and treatment algorithms are not always applicable or appropriate (ie, use of hydroxyurea).

\section{JAK Inhibition in MF}

Demonstration of universal JAK-STAT dysregulation in $\mathrm{MPN}^{67}$ supports JAK inhibition as a useful therapeutic strategy, especially for patients with MF, regardless of mutational status. Ruxolitinib was the first JAK inhibitor to be approved for the treatment of MF, based on 2 randomized studies demonstrating a $35 \%$ or more reduction in spleen volume compared with placebo (COMFORT-I, $41.9 \%$ vs $0.7 \%$ at 24 weeks; $P<.001)$ and best available therapy $(\mathrm{COM}$ FORT-II, $28.5 \%$ vs $0 \%$ at 48 weeks; $P<.001){ }^{68,69}$ Further, ruxolitinib resulted in clinically meaningful improvements in quality of life and the MF symptom burden. Specifically, as assessed by a validated tool, a $50 \%$ or more improvement in baseline symptoms was seen in $45.9 \%$ of patients compared with $5.3 \%$ in the ruxolitinib versus placebo arm $(P<.001){ }^{68}$

Grades 3 and 4 anemia and thrombocytopenia were reported in $45.2 \%$ and $12.9 \%$ of patients treated with ruxolitinib, respectively, in COMFORT-I, ${ }^{68}$ and grade 3 or 4 anemia ( $34 \%$ and $8 \%$, respectively) and thrombocytopenia ( $6 \%$ and $2 \%$, respectively) were reported in COMFORT-II. ${ }^{69}$ Nonhematologic adverse events included dizziness, headache, and easy bruising. ${ }^{68}$

The 2- and 3-year follow-up data from the COMFORT studies suggest durable responses. ${ }^{70,71}$ Responses seem to be independent of JAK2 ${ }^{\mathrm{V} 617 \mathrm{~F}}$ status, because recently JAK inhibitor responses were reported in CALR-mutated MF.72 Survival benefits have also been reported in patients treated with ruxolitinib compared with those treated with placebo or best available therapy and historical controls, including those from the DIPSS cohort. ${ }^{70,71,73,74}$ With a longer follow-up duration, the magnitude of this survival benefit has decreased, perhaps because of a crossover effect. The survival benefit attributed to ruxolitinib may be due to improvements in physical function and performance from amelioration of splenomegaly and reduction in cytokine levels, rather than to a direct anticlonal effect or changes in bone marrow histopathology.

Data are emerging on the use of ruxolitinib before transplant, and a clinical trial from the MPD Research Consortium (ClinicalTrials.gov identifier: NCT01790295) will also address this issue. Other JAK inhibitors are being studied in phase III trials, including pacritinib (ClinicalTrials.gov identifiers: NCT02055781 and NCT01773187) and momelotinib (ClinicalTrials.gov identifiers: NCT01969838 and NCT02101268). Pacritinib appears to be nonmyelosuppressive, ${ }^{75}$ and momelotinib ${ }^{76}$ may improve the hemoglobin and/or red blood cell transfusion dependence. These findings require confirmation in phase III studies.

\section{A New Role for JAK Inhibitor Therapy in PV}

A prior study reported hydroxyurea resistance and intolerance in $11 \%$ and $13 \%$ of patients with PV, respectively, and the former was associated with a higher risk of death and disease transformation. ${ }^{77}$ JAK inhibitors may have a role in this patient population. Phase II data for ruxolitinib given at $10 \mathrm{mg}$ twice daily revealed a complete response in $59 \%$ of patients, with thrombocytopenia as the most common adverse event. ${ }^{78}$ Subsequently, a phase III study compared ruxolitinib with best available therapy in patients with hydroxyurea-resistant/intolerant disease with splenomegaly and an ongoing phlebotomy requirement. ${ }^{79}$ Significantly more patients in the ruxolitinib arm achieved the primary end point of phlebotomy independence and spleen volume reduction at week $32(21 \%$ vs $1 \% ; P<.0001)$. When evaluating each component individually, 38\% and 60\% in the ruxolitinib arm achieved spleen volume reduction and hematocrit control compared with $1 \%$ and $20 \%$ in the best available therapy arm, respectively. In addition, significant improvements in baseline PV symptoms (cytokine-mediated, hyperviscosity, and spleen-related) as assessed using the MPN-SAF were noted in patients treated with ruxolitinib versus best available therapy.

Although the study was not powered to detect any difference in thrombosis rates, this adverse effect was reported in 1 patient in the ruxolitinib arm and 6 patients in the best available therapy arm. Grade 3 or 4 anemia and thrombocytopenia occurred in 1.8\% 
and $5.5 \%$ of ruxolitinib-treated patients, respectively. Diarrhea, muscle spasms, dizziness, and dyspnea were more commonly noted in the ruxolitinib arm. ${ }^{79}$ Based on these results, the FDA approved ruxolitinib for use in patients with inadequate response or intolerance to hydroxyurea. The safety and efficacy of momelotinib will also be evaluated in patients with ET and PV (ClinicalTrials.gov identifier: NCT01998828).

\section{Monitoring Therapy, Judging Net Effect, and Recognizing Disease Progression}

An evaluation of the efficacy of MPN therapy involves assessment of subjective symptom relief and objective parameters through palpation of the spleen (or with ultrasound/CT if needed) and CBC count monitoring. Although molecular monitoring is paramount in assessing response in chronic myelogenous leukemia (CML), measurement of the JAK2 $2^{\mathrm{V} 617 \mathrm{~F}}$ allelic burden is not currently recommended for widespread use in clinical practice. Routine, serial bone marrow biopsies are not typically indicated outside of clinical trials. Ruxolitinib has been shown to impair dendritic cell function, ${ }^{80}$ which may explain both positive and negative consequences of an anti-inflammatory medication. Case reports exist of opportunistic infections such as progressive multifocal leukoencephalopathy, ${ }^{81}$ retinal toxoplasmosis, ${ }^{82}$ tuberculosis, ${ }^{83,84}$ and cryptococcal pneumonia ${ }^{85}$ in ruxolitinib-treated patients. Abrupt discontinuation of therapy also presents a potential for severe rebound symptoms.

Tyrosine kinase inhibitor therapy has had a remarkable impact on preventing disease transformation in CML, but currently progression is an expected consequence for BCR-ABL-negative MPN. In part, progression can be reflected by a change in the symptomology, because onset of constitutional symptoms and progression of splenomegaly heralds MF transformation in patients with ET or PV. ${ }^{86}$ Criteria for this transformation also include development of anemia, leukoerythroblastosis, and bone marrow fibrosis. ET, PV, and MF can accelerate to an MPN blast phase, and consensus criteria for this transformation also exist. ${ }^{86}$

\section{Supportive Care: Symptoms, Cytopenias, and Splenomegaly}

Supportive needs include management of microvascular symptoms, such as migraines, atypical transient ischemic attack, visual disturbance, and erythromelalgia, which are often relieved by aspirin. ${ }^{87}$ Fatigue is prevalent; treatment strategies for this symptom are challenging, but nonpharmacologic options have been attempted. ${ }^{7}$ Pruritus can be debilitating, and treatments vary from antihistamines, paroxetine, interferon-alpha, and ultraviolet light therapy to JAK inhibitors.

Treatment for MF-associated anemia is an unmet need, ${ }^{88}$ but conventional agents can be of modest benefit. Conventional strategies have included androgens, erythropoietin-stimulating agents, and immunomodulatory drugs such as thalidomide or lenalidomide. The presence of transfusion-dependence, abnormal cytogenetics, increased erythropoietin levels, splenomegaly comorbidities (neuropathy), and other cytopenias can negatively impact tolerability or efficacy of these agents. Evidence of iron overload in patients with MF, particularly in those with hepatic or cardiac dysfunction, may have major implications for subsequent morbidity, but the use of parenteral or oral iron chelators in these settings needs prospective evaluation. ${ }^{89}$ The role of thrombopoietic agents (romiplostim, eltrombopag) to treat thrombocytopenia requires further study, given their potential to increase marrow fibrosis. ${ }^{90}$

Among the myeloid neoplasms, massive splenomegaly is unique to MF, and for some patients, medical therapy is inadequate. Often, splenectomy or splenic radiation are proposed; however, given the associated morbidity and mortality ${ }^{91}$ and the utility of JAK inhibitors in reducing marked symptomatic splenomegaly, providers must carefully select optimal candidates and suggest strategies to reduce perioperative complications, including use of prophylaxis or hydroxyurea to lessen risk of AVT and severe thrombocytosis.

Radiation therapy was shown to be useful for nonhepatosplenic extramedullary hematopoiesis $(\mathrm{EMH}) .{ }^{92}$ In a retrospective study, low-dose radiation therapy was used for spinal EMH (median dose of $1 \mathrm{~Gy}$; range, 1-10 Gy), pleural or pulmonary EMH (median dose of 1.25 Gy; range, 1.00-1.50 Gy), and abdominal or pelvic EMH (median dose of 2.02 Gy; range, $1.50-4.50 \mathrm{~Gy}$ ).

\section{The Role and Timing of Hematopoietic Stem Cell Transplantation}

Hematopoietic stem cell transplantation (HSCT) represents the only potential cure and is typically restricted to highly selected patients with $\mathrm{MF}$, given 
Evolving Management Strategies for Myeloproliferative Neoplasms

the risk-benefit ratio. In part, decision-making is based on risk-assessment; currently, the risk of transplant may be justified in patients with intermediate-2 or high risk disease, as assessed by IPSS or DIPSS/ DIPSS plus. In a retrospective series of 289 patients undergoing transplant between 1989 and 2002, transplant-related mortality (TRM) at day 100 was $22 \%$ and $42 \%$, respectively, for those with matched sibling donor (MSD) and matched unrelated donor (MUD). ${ }^{93}$ The overall survival rates at 5 years were $39 \%$ and $31 \%$ for MSD and MUD, respectively.

Outcomes were slightly improved in 162 patients undergoing reduced-intensity conditioning transplants from 1999 to 2009, with a 22\% TRM at 1 year and $62 \%$ overall survival at 5 years. ${ }^{94} \mathrm{~A}$ larger study of reduced-intensity conditioning transplants $(\mathrm{N}=233)$ reported a 5 -year overall survival rate of $47 \%$ (56\% for MSD, 48\% with MUD). ${ }^{95}$ A recent prospective study of 66 patients undergoing reduced-intensity conditioning reported on the strong influence of donor type, because overall survival and nonrelapse mortality rates were $75 \%$ and $22 \%$ in the MSD group, compared with $32 \%$ and $59 \%$ in the MUD group. ${ }^{96}$ Splenectomy before HSCT is not recommended given the associated morbidity, mortality, and lack of clear benefit. ${ }^{97}$ The role of JAK inhibitors before HSCT is still being defined in clinical trials. Interestingly, JAK inhibition after transplant may be useful for the treatment of acute graft versus host disease. ${ }^{98}$

\section{Additional Novel Therapies}

JAK inhibition may address symptoms and splenomegaly and has improved survival in MF; however, complete remission is not expected, resistance can develop because of heterodimer formation with other JAKs, and responses are rapidly lost with drug discontinuation. ${ }^{99}$ JAK inhibitors may serve as a foundation to which novel therapies may be added, however. As with most hematologic malignancies, combination strategies may further impact the natural history of MF (Figure 1). Several novel companions to JAK inhibitors are being evaluated in clinical trials, particularly in transplant-ineligible patients.

In early $\mathrm{MF}$, there is renewed interest in using interferon-alpha (ClinicalTrials.gov identifier: NCT01758588) based on smaller studies showing impressive response rates, including a minority of patients experiencing histologic improvements. ${ }^{100} \mathrm{Al}$ though neither has an established role in early or lowrisk MF, combining pegylated interferon and a JAK inhibitor represents a novel concept. In patients with anemia, adding a conventional agent to JAK inhibitors may offset myelosuppression. Danazol (ClinicalTrials.gov identifier: NCT01732445) has been added to ruxolitinib, and although combination therapy was well tolerated (no incremental toxicity with danazol), only 1 patient experienced clinical improvement. ${ }^{101}$ The combination of immunomodulatory drugs such as lenalidomide (ClinicalTrials.gov identifier: NCT01375140) or pomalidomide (ClinicalTrials.gov identifier: NCT01644110) and JAK inhibitors has also been preliminarily reported, with poor tolerability of the former combination when used concurrently, and relative safety of the latter, noting a sample size of 6 patients. ${ }^{102,103}$

In accelerated MF or blast phase MPN, a novel approach involves the use of hypomethylating agents (decitabine or 5-azacytidine; ClinicalTrials.gov identifiers: NCT02076191 and NCT01787487, respectively) with a JAK inhibitor. Other unique and recently reported combinations include the addition of histone deacetylase inhibitors (ClinicalTrials.gov identifiers: NCT01693601 and NCT01433445), PI3K inhibitors (ClinicalTrials.gov identifier: NCT01730248), and hedgehog signaling inhibitors (ClinicalTrials.gov identifier: NCT01787552) to ruxolitinib; the combinations seem to be tolerable and have shown impressive (preliminary) splenomegaly responses. ${ }^{104-106}$ JAK inhibitors paired with mTOR inhibitors, aurora kinase $\mathrm{A}$ inhibitors, and heat shock protein 90 inhibitors are also rational combinations. ${ }^{107}$

PRM-151, a recombinant form of pentraxin-2 and novel antifibrotic agent, was tolerable alone or with ruxolitinib, and despite a lower overall response rate, trended toward improvement in anemia and thrombocytopenia and bone marrow fibrosis grade (ClinicalTrials.govidentifier:NCT01981850). ${ }^{108}$ Finally, the telomerase inhibitor imetelstat (ClinicalTrials.gov identifier: NCT01731951) was myelosuppressive, leading to grade 4 neutropenia (18\%) and thrombocytopenia $(21 \%)$. However, treatment resulted in 4 complete responses and 3 partial responses, justifying further study in an expanded clinical trial. ${ }^{109}$

\section{Conclusions}

The discovery of JAK2 $2^{\mathrm{V} 61 \mathrm{~F}}$ has ushered in a new era for the MPN field, characterized by identification of recurring molecular aberrations such $J A K 2^{\mathrm{V} 617 \mathrm{~F}}$ and 
CALR mutations. This era has raised awareness of the impact of the MPN symptom burden, and prognostic tools have become increasingly sophisticated. The discovery of JAK-STAT dysregulation has been rapidly translated into novel therapeutic strategies for MF, with the JAK inhibitor ruxolitinib receiving approval and several additional agents in this drug class currently in advanced stages of clinical development. Specific targeting of CALR-mutant MF is also an intriguing concept. Although improvement in symptoms and splenomegaly is clearly important, novel strategies will hopefully result in anticlonal activity, improved bone marrow histopathology, and ultimately, complete remissions. The rapid pace and abundance of these developments are both overwhelming and encouraging for hematologists/oncologists and patients. The hope is that this MPN era will see significant alterations in natural history and improved clinical outcomes, as has occurred with Dameshek's other "MPD," CML.

\section{References}

1. Tefferi A. The history of myeloproliferative disorders: before and after Dameshek. Leukemia 2008;22:3-13.

2. Dameshek W. Some speculations on the myeloproliferative syndromes. Blood 1951;6:372-375.

3. James C, Ugo V, Le Couedic JP, et al. A unique clonal JAK2 mutation leading to constitutive signalling causes polycythaemia vera. Nature 2005;434:1144-1148.

4. Mehta J, Wang H, Iqbal SU, Mesa R. Epidemiology of myeloproliferative neoplasms in the United States. Leuk Lymph 2014;55:595-600.

5. Price GL, Davis KL, Karve S, et al. Survival patterns in United States (US) medicare enrollees with non-CML myeloproliferative neoplasms (MPN). PLoS One 2014:9:e90299.

6. Geyer HL, Emanuel RM, Dueck AC, et al. Distinct clustering of symptomatic burden amongst myeloproliferative neoplasm patients: retrospective assessment in 1470 patients. Blood 2014;12;123:3803-3810.

7. Mesa RA, Niblack J, Wadleigh M, et al. The burden of fatigue and quality of life in myeloproliferative disorders (MPDs): an international Internet-based survey of 1179 MPD patients. Cancer 2007;109:68-76.

8. Scherber R, Dueck AC, Johansson P, et al. The Myeloproliferative Neoplasm Symptom Assessment Form (MPN-SAF): international prospective validation and reliability trial in 402 patients. Blood 2011;118:401-408.

9. Emanuel RM, Dueck AC, Geyer HL, et al. Myeloproliferative neoplasm (MPN) symptom assessment form total symptom score: prospective international assessment of an abbreviated symptom burden scoring system among patients with MPNs. J Clin Oncol 2012;30:4098-4103.

10. Cervantes F, Dupriez B, Pereira A, et al. New prognostic scoring system for primary myelofibrosis based on a study of the International Working Group for Myelofibrosis Research and Treatment. Blood 2009;113:2895-2901.

11. Passamonti F, Cervantes F, Vannucchi AM, et al. A dynamic prognostic model to predict survival in primary myelofibrosis: a study by the IWG-MRT (International Working Group for Myeloproliferative Neoplasms Research and Treatment). Blood 2010;115:1703-1708.

12. Gangat N, Caramazza D, Vaidya R, et al. DIPSS plus: a refined Dynamic International Prognostic Scoring System for primary myelofibrosis that incorporates prognostic information from karyotype, platelet count, and transfusion status. J Clin Oncol 2011;29:392-397.

13. Pikman $Y$, Lee BH, Mercher T, et al. MPLW515L is a novel somatic activating mutation in myelofibrosis with myeloid metaplasia. PLoS Med 2006;3:e270
14. Pardanani AD, Levine RL, Lasho $\mathrm{T}$, et al. MPL515 mutations in myeloproliferative and other myeloid disorders: a study of 1182 patients. Blood 2006;108:3472-3476.

15. Scott LM, Tong W, Levine RL, et al.JAK2 exon 12 mutations in polycythemia vera and idiopathic erythrocytosis. N Engl J Med 2007;356:459-468.

16. Spolverini A, Pieri L, Guglielmelli P, et al. Infrequent occurrence of mutations in the PH domain of LNK in patients with JAK2 mutationnegative 'idiopathic' erythrocytosis. Haematologica 2013;98:e101-102.

17. Vainchenker W, Delhommeau F, Constantinescu SN, Bernard OA. New mutations and pathogenesis of myeloproliferative neoplasms. Blood 2011;118:1723-1735.

18. Klampfl T, Gisslinger H, Harutyunyan AS, et al. Somatic mutations of calreticulin in myeloproliferative neoplasms. N Engl J Med 2013;369:2379_ 2390.

19. Nangalia J, Massie CE, Baxter EJ, et al. Somatic CALR mutations in myeloproliferative neoplasms with nonmutated JAK2. N Engl J Med 2013;369:2391-2405.

20. Broseus J, Park JH, Carillo S, et al. Presence of calreticulin mutations in JAK2-negative polycythemia vera. Blood 2014:124:3964-3966.

21. Rumi E, Pietra D, Pascutto $C$, et al. Clinical effect of driver mutations of JAK2, CALR, or MPL in primary myelofibrosis. Blood 2014;124:1062-1069.

22. Tefferi A, Guglielmelli P, Larson DR, et al. Long-term survival and blast transformation in molecularly-annotated essential thrombocythemia, polycythemia vera and myelofibrosis. Blood 2014;124:2507-2513; quiz 2615

23. Tefferi A, Lasho TL, Tischer A, et al. The prognostic advantage of calreticulin mutations in myelofibrosis might be confined to type 1 or type 1-like CALR variants. Blood 2014;124:2465-2466

24. Vannucchi AM, Lasho TL, Guglielmelli P, et al. Mutations and prognosis in primary myelofibrosis. Leukemia 2013;27:1861-1869.

25. Tefferi A, Thiele J, Orazi A, et al. Proposals and rationale for revision of the World Health Organization diagnostic criteria for polycythemia vera, essential thrombocythemia, and primary myelofibrosis: recommendations from an ad hoc international expert panel. Blood 2007;110:1092-1097.

26. Kiladjian JJ, Cervantes F, Leebeek FW, et al. The impact of JAK2 and MPL mutations on diagnosis and prognosis of splanchnic vein thrombosis: a report on 241 cases. Blood 2008;111:4922-4929.

27. Spivak JL, Silver RT. The revised World Health Organization diagnostic criteria for polycythemia vera, essential thrombocytosis, and primary myelofibrosis: an alternative proposal. Blood 2008;112:231-239.

28. Barbui T, Thiele J, Passamonti F, et al. Survival and disease progression in essential thrombocythemia are significantly influenced by accurate morphologic diagnosis: an international study. J Clin Oncol 2011;29:3179_ 3184.

29. Kvasnicka HM, Thiele J. Prodromal myeloproliferative neoplasms: the 2008 WHO classification. Am J Hematol 2010;85:62-69.

30. Wilkins BS, Erber WN, Bareford D, et al. Bone marrow pathology in essential thrombocythemia: interobserver reliability and utility for identifying disease subtypes. Blood 2008;111:60-70.

31. Barbui $\mathrm{T}$, Thiele J, Gisslinger $\mathrm{H}$, et al. Masked polycythemia vera (mPV): results of an international study. Am J Hematol 2014;89:52-54.

32. Barbui $\mathrm{T}$, Thiele J, Vannucchi AM, Tefferi A. Rethinking the diagnostic criteria of polycythemia vera. Leukemia 2013;28:1191-1195.

33. Barbui T, Thiele J, Carobbio A, et al. Masked polycythemia vera diagnosed according to WHO and BCSH classification. Am J Hematol 2014;89:199_ 202.

34. Silver RT, Chow W, Orazi A, et al. Evaluation of WHO criteria for diagnosi of polycythemia vera: a prospective analysis. Blood 2013;122:1881-1886.

35. Cassinat B, Laguillier C, Gardin C, et al. Classification of myeloproliferative disorders in the JAK2 era: is there a role for red cell mass? Leukemia 2008;22:452-453.

36. Alvarez-Larran A, Ancochea A, Angona A, et al. Red cell mass measurement in patients with clinically suspected diagnosis of polycythemia vera or essential thrombocythemia. Haematologica 2012;97:1704-1707.

37. Tefferi A, Thiele J, Vannucchi AM, Barbui T. An overview on CALR and CSF3R mutations and a proposal for revision of WHO diagnostic criteria for myeloproliferative neoplasms. Leukemia 2014;28:1407-1413.

38. Barbui T, Finazzi G, Carobbio A, et al. Development and validation of an International Prognostic Score of thrombosis in World Health Organization: essential thrombocythemia (IPSET-thrombosis). Blood 2012;120:51285133; quiz 252.

39. Landolfi R, Marchioli R, Kutti J, et al. Efficacy and safety of low-dose aspirin in polycythemia vera. $\mathrm{N}$ Engl J Med 2004;350:114-124. 
Evolving Management Strategies for Myeloproliferative Neoplasms

40. Passamonti F, Rumi E, Pungolino E, et al. Life expectancy and prognostic factors for survival in patients with polycythemia vera and essential thrombocythemia. Am J Med 2004;117:755-761.

41. Barbui $T$, Carobbio $A$, Rumi $E$, et al. In contemporary patients with polycythemia vera, rates of thrombosis and risk factors delineate a new clinical epidemiology. Blood 2014;124:3021-3023.

42. Tefferi A, Rumi E, Finazzi G, et al. Survival and prognosis among 1545 patients with contemporary polycythemia vera: an international study. Leukemia 2013;27:1874-1881.

43. Stein BL, Saraf S, Sobol U, et al. Age-related differences in disease characteristics and clinical outcomes in polycythemia vera. Leuk Lymph 2013;54:1989-1995.

44. Falanga A, Marchetti M. Thrombosis in myeloproliferative neoplasms. Semin Thrombosis Hemostasis 2014;40:348-358.

45. Rumi E, Pietra D, Ferretti V, et al. JAK2 or CALR mutation status defines subtypes of essential thrombocythemia with substantially different clinical course and outcomes. Blood 2014;123:1544-1551.

46. Rotunno G, Mannarelli C, Guglielmelli P, et al. Impact of calreticulin mutations on clinical and hematological phenotype and outcome in essential thrombocythemia. Blood 2014;123:1552-1555.

47. Barbui T, Barosi G, Birgegard G, et al. Philadelphia-negative classical myeloproliferative neoplasms: critical concepts and management recommendations from European LeukemiaNet. J Clin Oncol 2011;29:761770.

48. Fruchtman SM, Mack K, Kaplan ME, et al. From efficacy to safety: a polycythemia vera study group report on hydroxyurea in patients with polycythemia vera. Semin Hematol 1997;34:17-23.

49. Cortelazzo S, Finazzi G, Ruggeri M, et al. Hydroxyurea for patients with essential thrombocythemia and a high risk of thrombosis. N Engl J Med 1995;332:1132-1136.

50. Harrison CN, Campbell PJ, Buck G, et al. Hydroxyurea compared with anagrelide in high-risk essential thrombocythemia. N Engl J Med 2005;353:33-45.

51. Campbell PJ, Scott LM, Buck G, et al. Definition of subtypes of essential thrombocythaemia and relation to polycythaemia vera based on JAK2 V617F mutation status: a prospective study. Lancet 2005;366:1945-1953.

52. Gisslinger H, Gotic M, Holowiecki J, et al. Anagrelide compared with hydroxyurea in WHO-classified essential thrombocythemia: the ANAHYDRET Study, a randomized controlled trial. Blood 2013;121:17201728 .

53. Sterkers Y, Preudhomme C, Lai JL, et al. Acute myeloid leukemia and myelodysplastic syndromes following essential thrombocythemia treated with hydroxyurea: high proportion of cases with $17 \mathrm{p}$ deletion. Blood 1998;91:616-622.

54. Spivak JL. An inconvenient truth. Blood 2010;115:2727-2728.

55. Kiladjian JJ, Chevret S, Dosquet C, et al. Treatment of polycythemia vera with hydroxyurea and pipobroman: final results of a randomized trial initiated in 1980. J Clin Oncol 2011;29:3907-3913.

56. Passamonti F, Thiele J, Girodon F, et al. A prognostic model to predict survival in 867 World Health Organization-defined essential thrombocythemia at diagnosis: a study by the International Working Group on Myelofibrosis Research and Treatment. Blood 2012;120:1197-1201.

57. Finazzi G, Ruggeri M, Rodeghiero F, Barbui T. Second malignancies in patients with essential thrombocythaemia treated with busulphan and hydroxyurea: long-term follow-up of a randomized clinical trial. $\mathrm{Br}$ J Haematol 2000;110:577-583

58. Kiladjian JJ, Cassinat B, Chevret $S$, et al. Pegylated interferon-alfa-2a induces complete hematologic and molecular responses with low toxicity in polycythemia vera. Blood 2008;112:3065-3072.

59. Quintas-Cardama A, Abdel-Wahab O, Manshouri T, et al. Molecular analysis of patients with polycythemia vera or essential thrombocythemia receiving pegylated interferon alpha-2a. Blood 2013;122:893-901.

60. Squizzato A, Romualdi E, Passamonti F, Middeldorp S. Antiplatelet drugs for polycythaemia vera and essential thrombocythaemia. Cochrane Database Syst Rev 2013;4:CD006503.

61. Pascale S, Petrucci G, Dragani A, et al. Aspirin-insensitive thromboxane biosynthesis in essential thrombocythemia is explained by accelerated renewal of the drug target. Blood 2012;119:3595-3603.

62. Marchioli R, Finazzi G, Specchia G, et al. Cardiovascular events and intensity of treatment in polycythemia vera. N Engl J Med 2013;368:22-33.

63. Carobbio A, Thiele J, Passamonti F, et al. Risk factors for arterial and venous thrombosis in WHO-defined essential thrombocythemia: an international study of 891 patients. Blood 2011;117:5857-5859.
64. Ruggeri M, Rodeghiero F, Tosetto A, et al. Postsurgery outcomes in patients with polycythemia vera and essential thrombocythemia: a retrospective survey. Blood 2008;111:666-671.

65. Teofili L, Giona F, Martini M, et al. Markers of myeloproliferative diseases in childhood polycythemia vera and essential thrombocythemia. J Clin Oncol 2007;25:1048-1053.

66. Giona F, Teofili L, Moleti ML, et al. Thrombocythemia and polycythemia in patients younger than 20 years at diagnosis: clinical and biologic features, treatment, and long-term outcome. Blood 2012;119:2219-2227.

67. Rampal R, Al-Shahrour F, Abdel-Wahab O, et al. Integrated genomic analysis illustrates the central role of JAK-STAT pathway activation in myeloproliferative neoplasm pathogenesis. Blood 2014;123:e123-133.

68. Verstovsek S, Mesa RA, Gotlib J, et al. A double-blind, placebo-controlled trial of ruxolitinib for myelofibrosis. N Engl J Med 2012;366:799-807.

69. Harrison C, Kiladjian JJ, Al-Ali HK, et al. JAK inhibition with ruxolitinib versus best available therapy for myelofibrosis. N Engl J Med 2012;366:787798.

70. Cervantes F, Vannucchi AM, Kiladjian JJ, et al. Three-year efficacy, safety, and survival findings from COMFORT-II, a phase 3 study comparing ruxolitinib with best available therapy for myelofibrosis. Blood 2013;122:4047-4053.

71. Verstovsek S, Mesa RA, Gotlib J, et al. Efficacy, safety and survival with ruxolitinib in patients with myelofibrosis: results of a median 2-year followup of COMFORT-I. Haematologica 2013;98:1865-1871.

72. Passamonti F, Caramazza D, Maffioli M. JAK inhibitor in CALR-mutant myelofibrosis. N Engl J Med 2014;370:1168-1169.

73. Verstovsek S, Kantarjian HM, Estrov Z, et al. Long-term outcomes of 107 patients with myelofibrosis receiving JAK1/JAK2 inhibitor ruxolitinib: survival advantage in comparison to matched historical controls. Blood 2012;120:1202-1209.

74. Passamonti F, Maffioli M, Cervantes F, et al. Impact of ruxolitinib on the natural history of primary myelofibrosis: a comparison of the DIPSS and the COMFORT-2 cohorts. Blood 2014;123:1833-1835.

75. Dean JP, Cernohous P, Komrokji RS, et al. Pacritinib, a dual JAK2/FLT3 inhibitor: an integrated efficacy and safety analysis of phase II trial data in patients with primary and secondary myelofibrosis (MF) and platelet counts $\leq 100,000 / \mu 1$. Presented at the 55th ASH Annual Meeting and Exposition; December 7-20, 2013; New Orleans, Louisiana. Abstract 395.

76. Pardanani A, Laborde RR, Lasho TL, et al. Safety and efficacy of CYT387, a JAK1 and JAK2 inhibitor, in myelofibrosis. Leukemia 2013;27:1322-1327.

77. Alvarez-Larran A, Pereira A, Cervantes F, et al. Assessment and prognostic value of the European LeukemiaNet criteria for clinicohematologic response, resistance, and intolerance to hydroxyurea in polycythemia vera. Blood 2012;119:1363-1369.

78. Verstovsek S, Passamonti F, Rambaldi A, et al. A phase 2 study of ruxolitinib, an oral JAK1 and JAK2 inhibitor, in patients with advanced polycythemia vera who are refractory or intolerant to hydroxyurea. Cancer 2014;120:513520.

79. Vannucchi AM, Kiladjian JJ, Griesshammer M, et al. Ruxolitinib versus standard therapy for the treatment of polycythemia vera. N Engl J Med 2015;372:426-435.

80. Heine A, Held SA, Daecke SN, et al. The JAK-inhibitor ruxolitinib impairs dendritic cell function in vitro and in vivo. Blood 2013;122:1192-1202.

81. Wathes R, Moule S, Milojkovic D. Progressive multifocal leukoencephalopathy associated with ruxolitinib. N Engl J Med 2013;369:197-198.

82. Goldberg RA, Reichel E, Oshry LJ. Bilateral toxoplasmosis retinitis associated with ruxolitinib. N Engl J Med 2013;369:681-683.

83. Hopman RK, Lawrence SJ, Oh ST. Disseminated tuberculosis associated with ruxolitinib. Leukemia 2014;28:1750-1751.

84. Colomba C, Rubino R, Siracusa L, et al. Disseminated tuberculosis in a patient treated with a JAK2 selective inhibitor: a case report. BMC Res Notes 2012;5:552.

85. Wysham NG, Sullivan DR, Allada G. An opportunistic infection associated with ruxolitinib, a novel janus kinase 1,2 inhibitor. Chest 2013;143:14781479.

86. Mesa RA, Verstovsek S, Cervantes F, et al. Primary myelofibrosis (PMF), post polycythemia vera myelofibrosis (post-PV MF), post essential thrombocythemia myelofibrosis (post-ET MF), blast phase PMF (PMF$\mathrm{BP}$ ): consensus on terminology by the international working group for myelofibrosis research and treatment (IWG-MRT). Leuk Res 2007;31:737740 .

87. Michiels JJ, Berneman Z, Schroyens W, et al. Platelet-mediated erythromelalgic, cerebral, ocular and coronary microvascular ischemic and thrombotic manifestations in patients with essential thrombocythemia and 
polycythemia vera: a distinct aspirin-responsive and coumadin-resistant arterial thrombophilia. Platelets 2006;17:528-544.

88. Guglielmelli P, Vannucchi AM. Struggling with myelofibrosis-associated anemia. Leuk Res 2013;37:1429-1431.

89. Elli EM, Belotti A, Aroldi A,et al. Iron chelation therapy with deferasirox in the management of iron overload in primary myelofibrosis. Mediterr J Hematol Infect Dis 2014;6:e2014042.

90. Ghanima W, Junker P, Hasselbalch HC, et al. Fibroproliferative activity in patients with immune thrombocytopenia (ITP) treated with thrombopoietic agents. Br J Haematol 2011;155:248-255.

91. Mesa RA, Nagorney DS, Schwager $S$, et al. Palliative goals, patient selection, and perioperative platelet management: outcomes and lessons from 3 decades of splenectomy for myelofibrosis with myeloid metaplasia at the Mayo Clinic. Cancer 2006;107:361-370.

92. Koch CA, Li CY, Mesa RA, Tefferi A. Nonhepatosplenic extramedullary hematopoiesis: associated diseases, pathology, clinical course, and treatment. Mayo Clin Proc 2003; 78:1223-1233.

93. Ballen KK, Shrestha S, Sobocinski KA, et al. Outcome of transplantation for myelofibrosis. Biol Blood Marrow Transplant 2010;16:358-367.

94. Alchalby $\mathrm{H}$, Badbaran $\mathrm{A}$, Zabelina $\mathrm{T}$, et al. Impact of JAK2V617F mutation status, allele burden, and clearance after allogeneic stem cell transplantation for myelofibrosis. Blood 2010;116:3572-3581.

95. Gupta V, Malone AK, Hari PN, et al. Reduced-intensity hematopoietic cell transplantation for patients with primary myelofibrosis: a cohort analysis from the center for international blood and marrow transplant research. Biol Blood Marrow Transplant 2014;20:89-97.

96. Rondelli D, Goldberg JD, Isola L, et al. MPD-RC 101 prospective study of reduced-intensity allogeneic hematopoietic stem cell transplantation in patients with myelofibrosis. Blood 2014;124:1183-1191.

97. Gupta V, Hari P, Hoffman R. Allogeneic hematopoietic cell transplantation for myelofibrosis in the era of JAK inhibitors. Blood 2012;120:1367-1379.

98. Spoerl S, Mathew NR, Bscheider M, et al. Activity of therapeutic JAK 1/2 blockade in graft-versus-host disease. Blood 2014;123:3832-3842.

99. Koppikar P, Bhagwat N, Kilpivaara O, et al. Heterodimeric JAK-STAT activation as a mechanism of persistence to JAK2 inhibitor therapy. Nature 2012;489:155-159.

100. Silver RT, Vandris K, Goldman JJ. Recombinant interferon-alpha may retard progression of early primary myelofibrosis: a preliminary report. Blood 2011;117:6669-6672.

101. Gowin KL, Dueck AC, Mascarenhas JO, et al. Interim analysis of a phase II pilot trial of ruxolitinib combined with danazol for patients with primary myelofibrosis (MF), post essential thrombocythemia-myelofibrosis (post ET), and post polycythemia vera myelofibrosis (PV MF) suffering from anemia.
Presented at the 56th ASH Annual Meeting and Exposition; December 6-9 2014; San Francisco, California. Abstract 3206.

102. Stegelmann F, Griesshammer M, Reiter A, et al. A multicenter phase-Ib/ II study of ruxolitinib/pomalidomide combination therapy in patients with primary and secondary myelofibrosis: safety data from the Mpnsg-0212 trial (NCT01644110). Presented at the 56th ASH Annual Meeting and Exposition; December 6-9, 2014; San Francisco, California. Abstract 3161.

103. Naval D, Cortes JE, Jabbour E, et al. Ruxolitinib and lenalidomide as a combination therapy for patients with myelofibrosis. Presented at the 56th ASH Annual Meeting and Exposition; December 6-9, 2014; San Francisco, California. Abstract 1831

104. Kiladjian JJ, Heidel FH, Vannucchi AM, et al. Efficacy, safety, and confirmation of the recommended phase 2 dose of ruxolitinib plus panobinostat in patients with intermediate or high-risk myelofibrosis. Presented at the 56th ASH Annual Meeting and Exposition; December 6-9 2014; San Francisco, California. Abstract 711.

105. Durrant S, Koren-Michowitz M, Lavie D, et al. HARMONY: an open-label multicenter, 2-arm, dose-finding, phase $1 \mathrm{~b}$ study of the combination of ruxolitinib and buparlisib (BKM120) in patients with myelofibrosis (MF) Presented at the 56th ASH Annual Meeting and Exposition; December 6-9, 2014; San Francisco, California. Abstract 710.

106. Gupta V, Koschmieder S, Harrison CN, et al. Phase $1 \mathrm{~b}$ dose-escalation study of sonidegib (LDE225) in combination with ruxolitinib (INC424) in patients with myelofibrosis. Presented at the 56th ASH Annual Meeting and Exposition; December 6-9, 2014; San Francisco, California. Abstract 712.

107. Stein BL, Swords R, Hochhaus A, Giles F. Novel myelofibrosis treatment strategies: potential partners for combination therapies. Leukemia 2014;28:2139-2147

108. Verstovsek S, Mesa RA, Foltz LM, et al. Phase 2 trial of PRM-151, an anti fibrotic agent, in patients with myelofibrosis: stage 1 results [abstract]. J Clin Oncol 2014;32(Suppl):Abstract 7114

109. Tefferi A, LaPlant BR, Begna $K$, et al. Imetelstat, a telomerase inhibitor, therapy for myelofibrosis: a pilot study. Presented at the 56th ASH Annual Meeting and Exposition; December 6-9, 2014; San Francisco, California. Abstract 634

110. Dupriez B, Morel P, Demory JL, et al. Prognostic factors in agnogenic myeloid metaplasia: a report on 195 cases with a new scoring system. Blood 1996;88:1013-1018

\title{
Last chance to earn CME/CE/CEU credit
}

Methotrexate and Fluorouracil Toxicities: ASCQ POST A Collaborative Practice Approach to Prevention and Treatment

\author{
Proceedings From a Roundtable Discussion, \\ January 26, 2014, St. Petersburg, Florida
}

\section{Featuring}

Lee S. Schwartzberg, MD, FACP

The West Clinic, Memphis, Tennessee
Wendy H. Vogel, MSN, FNP, AOCNP® Wellmont Cancer Institute. Kingsport, Tennessee
Christopher J. Campen, PharmD, BCPS, BCOP

The University of Arizona

Cancer Center, Tucson, Arizona
News and views from the world of clinical oncology and hematology

Methotrexate and Fluorouracil Toxicities: A Collaborative Practice Approach to Prevention and Treatment

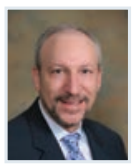

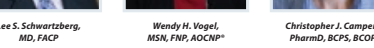

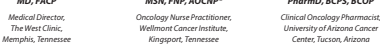

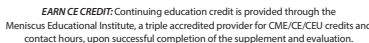
ASCO

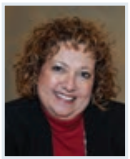

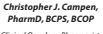

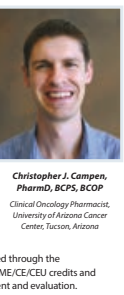

Editor-in-Chief, James O.Ammitage, MD | Ascopost.com 